\title{
Wave Characteristics of Falling Film on Inclination Plate at Moderate Reynolds Number
}

\author{
Chuan Lu, Sheng-Yao Jiang, and Ri-Qiang Duan \\ Institute of Nuclear and New Energy Technology, Collaborative Innovation Center of Advanced Nuclear Energy Technology, \\ Key Laboratory of Advanced Reactor Engineering and Safety of Ministry of Education, Tsinghua University, Beijing, China
}

Correspondence should be addressed to Ri-Qiang Duan; duanrq@mail.tsinghua.edu.cn

Received 20 June 2016; Accepted 10 August 2016

Academic Editor: Xiangdong Li

Copyright (C) 2016 Chuan Lu et al. This is an open access article distributed under the Creative Commons Attribution License, which permits unrestricted use, distribution, and reproduction in any medium, provided the original work is properly cited.

Falling water film on an inclined plane is studied by shadowgraphy. The ranges of inclination angle and the film Reynolds number are, respectively, up to $21^{\circ}$ and 60 . Water is used as working fluid. The scenario of wave regime evolution is identified as three distinctive regimes, namely, initial quiescent smooth film flow, two-dimensional regular solitary wave pattern riding on film flow, and three-dimensional irregular wave pattern. Three characteristic parameters of two-dimensional solitary wave pattern, namely, inception length, primary pulse spacing, and propagation velocity, are examined, which are significant in engineering applications for estimation of heat and mass transfer on film flow. The present experimental data are well in agreement with the Koizumi correlations, the deviation from which is limited to $20 \%$ and $15 \%$, respectively, for primary pulse spacing and propagation velocity. Through the scrutiny of the present experimental observation, it is concluded that wave evolution on film flow at the moderate Reynolds number is controlled by gravity and drag and the Rayleigh-Taylor instability that occurred on the steep front of primary pulse triggers the disintegration of continuous two-dimensional regular solitary wave pattern into three-dimensional irregular wave pattern.

\section{Introduction}

Falling thin film flow has special applications in nuclear engineering due to its unique advantages, namely, high thermal efficiency, zero pumping power consumption, and low flow rate. For AP1000 nuclear plants, film flow falling down from a storage tank is the core safety measure of the Passive Cooling Containment System (PCCS). In seawater thermal desalination powered by nuclear energy, pure water is produced via evaporation of film flow attached to tube-bundle surface in multiple-effect evaporators due to its high thermal efficiency. To meet the demand of engineering applications, Yu and Cheng [1] investigated statistically falling film on large-scale plates to develop an empirical correlation among flowrate, film thickness, and wave characteristics. However, as well known, the behaviors of heat and mass transfer of film flow are more dependent on film topography and surface wave dynamics than substrate layer flow. Thus, in this paper, flow pattern and wave characteristics on film flow are experimentally scrutinized to explore the surface topological change for evaluation of heat and mass transfer on film flow from the physical viewpoint.

The wave evolution of falling film is mainly controlled by the Reynolds number, which Portalski [2] used to classify wave mode of falling film flow. Our experiments cover the film Reynolds number in the moderate range from 10 to 60 , the corresponding wave modes under which span pseudolaminar up to pseudoturbulent regime [2]. Film flow at the moderate Reynolds number has many applications in industry [3]. At the moderate Reynolds number, it has been established that waves, appearing on a smooth surface, develop quickly into two-dimensional ordered solitary wave pattern featured by the primary pulse preceding with a series of small capillary ripples, and then the pattern is readily susceptible to precipitate breakup into three-dimensional chaotic wave pattern [4]. To discern wave structure and characteristics, small inclination angle (up to $20^{\circ}$ ) is used in this experiments to slow down its spacial variation. 
In this present experiments, initial disturbance of falling film flows is imposed in the natural excitation way. Twodimensional solitary wave riding on film flow is investigated in this study, including its dynamics, interaction with each other, and transition to three-dimensional complex disordered wave pattern. Two-dimensional solitary wave on film flow was first recorded in the P. L. Kapitza and S. P. Kapitza's pioneering work [5]. It exhibits rich information of coherent structure and nonlinear hydrodynamics of wavy film flow and intrigues a lot of theoretical and numerical investigations on its behaviors and characteristics. Most of previous experimental researches of two-dimensional solitary wave on film flow $[4,6]$ were limited on forcing excitation by introducing single-frequency disturbance at film entrance, which is however different from practical scenarios of film flow due to the linear filtering nature and the effect of inescapable surrounding noise. Because instabilities of film flow are noise-sustained [7], falling film flow dynamics are considerately affected by the inlet conditions. Natural disturbances (white noise) introduced into inlet evolve on film flow with different scenarios from artificially introduced excitation in inlet. The study of our present experiment is carried out under natural excitation, which is meaningful for engineering applications, as mentioned above in the PCCS of the AP1000 nuclear reactor and the multiple-effect evaporator in seawater desalination. Meanwhile, the previous experimental works on two-dimensional solitary wave on film flow mostly focused on variation of local film thickness [8,9]. In this study, two key parameters of two-dimensional solitary wave on film flow, namely, primary pulse spacing (wave length) and its propagation velocity, are paid attention to, which are significant to estimate heat and mass transfer for film flow.

On the triggering mechanism of transition of twodimensional ordered solitary pattern into disordered threedimensional pattern, it has not yet got well elucidated and no agreement has been achieved. Liu et al. [3] roughly redefined the transition as a secondary instability, which is assumed to initiate coalescence, and splitting processes of twodimensional solitary wave fronts and furthermore evolve into complicated three-dimensional disordered patterns. Park and Nosoko [10] conjectured that the capillary instability (the Plateau-Rayleigh instability) occurring at the valley right next to primary solitary pulse triggers the transition due to its large curvature, which results in necking breakup of solitary pulse. So there is need to clarify the mechanism in this study. Furthermore, all the previous works on the transition issue are addressed to forcing excitation in inlet. In this paper, the transition to three-dimensional instability on film flow under natural disturbance in inlet is examined, which is motivated by the noise-sustained behavior of film flow instability.

In the present experiments, distilled water is used as working fluid. The shadowgraphic technique is employed to visualize wave structure and flow pattern and measure variables over liquid film. The method is low-cost and highsensitive to film thickness. It can obtain readily the detail contour of film topography.

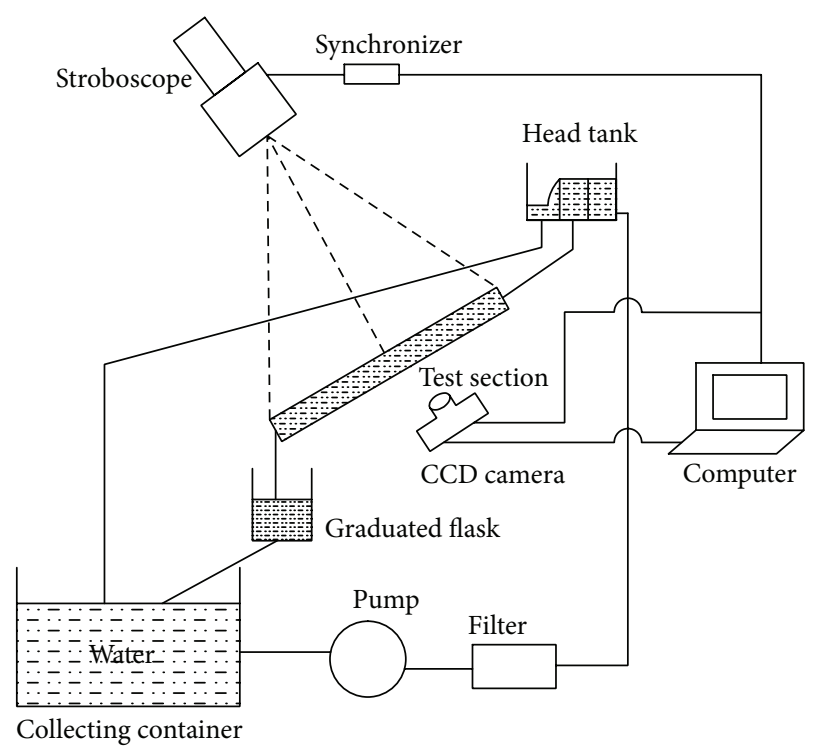

FIGURE 1: Schematic diagram of experimental system.

\section{Experiment Apparatus and Procedure}

The experimental system used in the present study is shown schematically in Figure 1. The experiments are conducted at room temperature and atmospheric pressure. Distilled water is pumped into a head tank through a filter to remove contamination. The head tank is constructed into three compartments to regulate the film Reynolds number and meanwhile isolate the disturbances out of pump vibration. The right compartment is linked to water feed from the filter. Water brims into the middle compartment and then further spills into the left draining compartment. In such a way, water level in the middle compartment is kept smooth and steady. The left one is used to drain the brimming water from the middle compartment. Water flows through a silicone tube, one end of which is linked to the bottom drain hole of the middle compartment, into a specially designed apparatus to produce water film flow. The film Reynolds number is regulated by adjusting the partition height of the middle compartment to change pressure head of film flow. The volumetric flow rate of film flow is measured with a flask and a stopwatch at the exit of the apparatus.

The apparatus for producing water film is schematically shown in Figure 2. The film plate is made of flat Plexiglas with $0.80 \mathrm{~m}$ in length and $0.30 \mathrm{~m}$ in width, which is fixed on a rectangular Plexiglas framework. To spread uniformly film flow on the film plate and minimize the effect of wetting difference along the film plate, a small portion is partitioned by a bar at the top of the film plate, in which multiple intertwined layers of $150 \mu$ sintered wire mesh with fabric thread are tightly stuffed. Water flows into the test section through the gap between the bar and the film plate. The apparatus is mounted on a supporting structure, which stands on a rubber pad to reduce any building vibration. The inclination angle can be continuously adjusted in the range of $0-45^{\circ}$ from horizontal plane. 


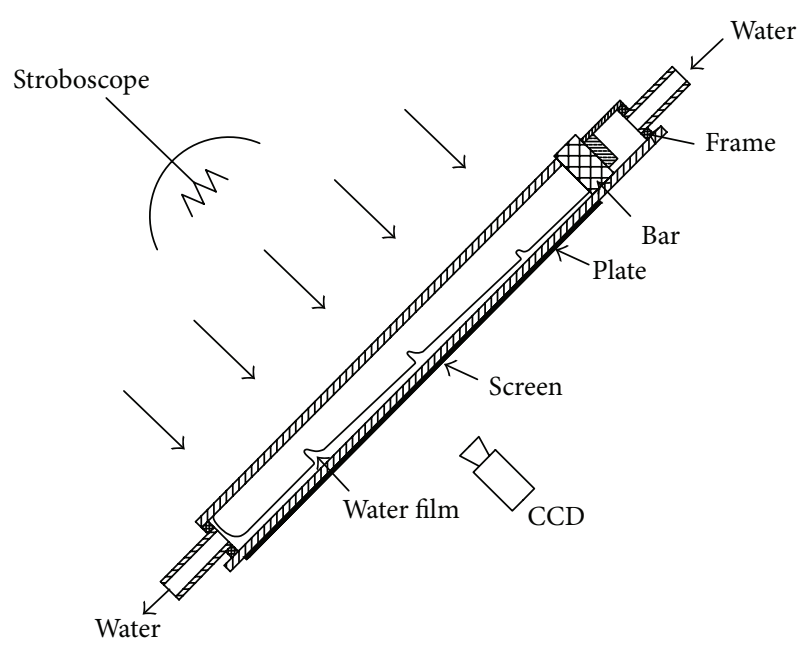

Figure 2: Apparatus of generating a falling water film.

Water film is illuminated with a stroboscope and its shadow on a screen is captured with a Charge Coupled Device (CCD) digital video camera synchronized with the stroboscope. The stroboscope flashes at the rate of 30-14000 FPM with $0.1 \mathrm{FPM}$ in both resolution and accuracy. Wave velocity is calculated from the displacement of the same wave peak and the time interval. The procedure is performed for a number of frames that are selected randomly. The average of measured values derived in this manner is defined as wave velocity for a certain experimental condition. The uncertainty of a measured wave velocity is less than approximately $0.0008 \mathrm{~m} / \mathrm{s}$. Wave separation is measured in a frame, in which at least two wave peaks are contained. As in wave velocity measurement, a number of frames are selected randomly. The average of measured values is taken as wave separation. The uncertainty of wave separation is less than $0.001 \mathrm{~m}$.

\section{Results and Discussion}

Film flow is investigated at the moderate Reynolds number from 10 to 60 . The experimental observations in the present study show wave on film flow in natural excitation is spatially evolved in three modes based on film topographical variation and wave pattern. In the entrance region of film flow, a train of equally spaced streaks appears on the surface of falling film, as shown in Figure 3, which is defined as the first mode. The lasting length of the first mode depends on the Reynolds number and the inclined angle of film plate. Then, wave evolution on film flow enters the second mode, featured with two-dimensional ordered solitary wave pattern, seen in Figure 4. The structure of solitary wave is characteristic of a large-amplitude primary pulse with steep front preceded with a series of capillary ripples, the amplitudes of which are decreased one by one downstream. Further downstream, two-dimensional ordered solitary wave pattern is precipitately transformed into three-dimensional irregular wave pattern, for example, discrete crater-like and caterpillar-like structures, as illustrated in Figure 5, which is defined as the third mode.

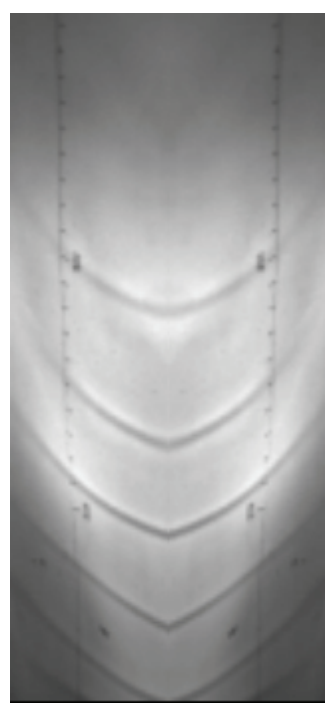

FIgURE 3: Regular streak-like wave pattern, $\beta=8^{\circ}$ and $\operatorname{Re}=22$.

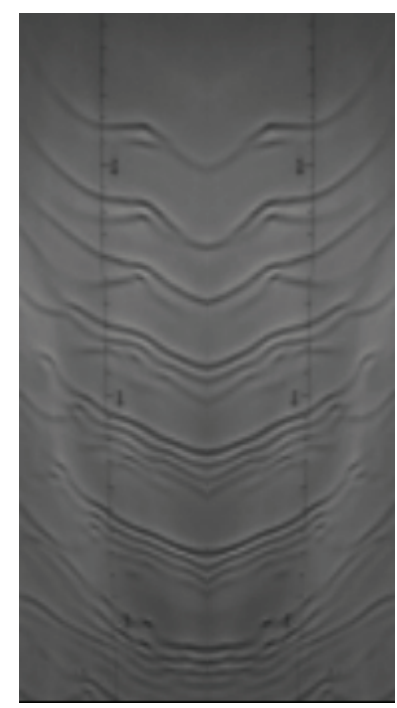

FIgURE 4: Two-dimensional regular wave packet, $\beta=13^{\circ}$ and $\operatorname{Re}=$ 32.

3.1. Spatial Development of Two-Dimensional Solitary. Wave evolution in two-dimensional solitary wave pattern is one significant stage of film flow in an inclined plate, which has great effect on heat transfer distribution. Two-dimensional solitary wave pattern starts appearing at a certain distance from the inlet and forms a train of solitary primary pulses downstream, which signals the inception of two-dimensional solitary wave pattern on film flow. They are almost equally spaced between each other with nearly flat film portion. Wave evolution in the two-dimensional solitary wave pattern is a typical convective instability, which is just modulated in the stream-wise direction and held stationary in the transverse direction. In our experiments, primary pulses are bulged out at the central plane due to sidewall effect, the shape of which is just reflection of the transverse distribution of the surface 


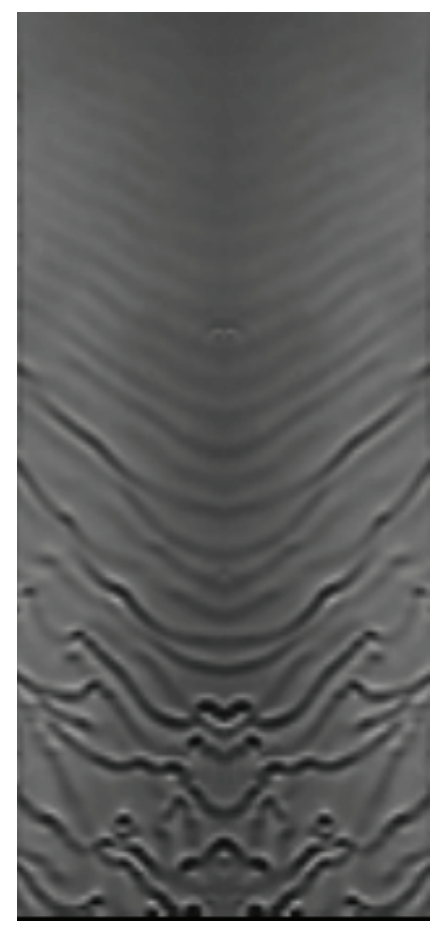

FIgURE 5: Two-dimensional regular wave packet, $\beta=13^{\circ}$ and $\mathrm{Re}=$ 56.

velocity. In this study, the following statistical qualities of two-dimensional solitary wave pattern are extracted, namely, inception length of two-dimensional solitary wave pattern, primary pulse spacing, and its propagation velocity, from the wave profile along the central stream-wise plane to diminish the sidewall effect.

Inception Length of Two-Dimensional Solitary. The inception length of two-dimensional solitary wave pattern is defined as the distance from the inlet where the first visible twodimensional solitary wave pattern can be recognized based on naked eye. In our investigation, the inception lengths of twodimensional solitary wave pattern fall into the range of $20 \mathrm{~cm}$ to $40 \mathrm{~cm}$, as shown in Table 1 , the values of which are consistent with those of Jones and Whitaker [11] observations (30$60 \mathrm{~cm}$ ) at the comparable inclination angle and the Reynolds number. The experimental results show that the inception length of solitary wave pattern is decreased with inclination angle and little dependent on the Reynolds number. So it is inferred that wave evolution at the moderate Reynolds number is still dominated by drag and gravity and the contribution of inertial force is not yet up to such a degree to change the global evolution trend of two-dimensional solitary wave.

Primary Pulse Spacing. Two-dimensional solitary wave pattern is regularly spaced in sequence with coherent structure. In our investigation, the average primary pulse spacing is in the range of $2-5 \mathrm{~cm}$, as shown in Figure 6 , the value of which falls into the range of gravity-driven wave and is in agreement with Jones and Whitaker [11] and Koizumi et al. [12] experiments. The experimental data reveal that the
TABLE 1: Inception length of two-dimensional solitary.

\begin{tabular}{lcc}
\hline $\begin{array}{l}\text { Inclination } \\
\text { angle }\end{array}$ & $\begin{array}{c}\text { Reynolds } \\
\text { number }\end{array}$ & $\begin{array}{c}\text { Inception length of } \\
\text { two-dimensional solitary }\end{array}$ \\
\hline $8^{\circ}$ & $21.2 \sim 28.3$ & $\sim 37$ \\
$13^{\circ}$ & $18.2 \sim 29.2$ & $\sim 26$ \\
$21^{\circ}$ & $56.7 \sim 57.1$ & $\sim 18.5$ \\
\hline
\end{tabular}

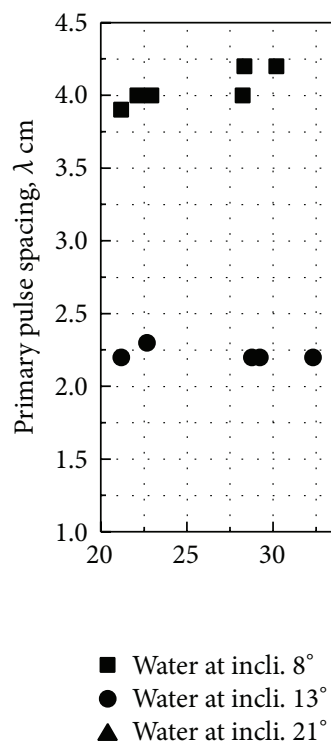

Figure 6: Separation spacing between adjacent solitary pulses.

dependence of the primary pulse spacing on inclination angle and the Reynolds number is the same as for inception length of solitary.

Solitary Wave Propagation Velocity. Due to riding on falling film flow, the propagation velocity of solitary wave results from both wave propagation itself and convection of surface film flow [13]. In this study, the propagation velocity is normalized with the Nusselt mean film velocity, as it is reduced to the ratio of $C_{w} / V_{\mathrm{Nu}}$, in part for manifestation of actual wave propagation and in part for comparison with the data of other researchers. The Nusselt mean film velocity is expressed as follows:

$$
V_{\mathrm{Nu}}=\frac{\rho g h_{\mathrm{Nu}}^{2}}{3 \mu},
$$

where $h_{\mathrm{Nu}}$ is Nusselt's flat film thickness for laminar falling film [2].

Figure 7 shows the ratio with the film Reynolds number at different inclination angle, the trend of which is consistent with previous experimental observations. The ratio exhibits a larger spread in the range of 2 to 3 at Re $\leq 30$, the value of which is in agreement with the prediction of Carey [14]. And the effect of inclination angle on wave propagation velocity appears almost independent of it at small Reynolds number. As the Reynolds number increases to $\sim 60$, the ratio asymptotically approaches a certain constant, the mechanism of which may be that the dependence of the ratio on the 


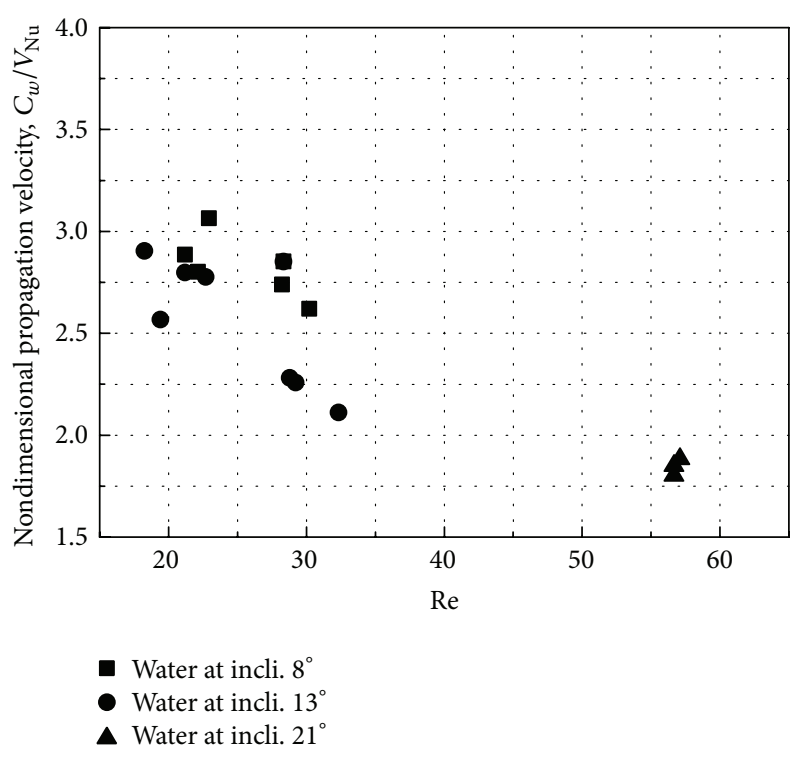

FIGURE 7: Solitary wave propagation velocity.

Reynolds number is the same as that for the Nusselt surface film velocity, causing the ratio to become independent on the Reynolds number.

Dimensionless Correlations of Primary Pulse Spacing and Propagation Velocity. Data reduction of primary pulse spacing and propagation velocity is performed based on the Buckingham $\pi$-theorem [15] in this study. For solitary wave on free film flow under natural excitation, primary pulse spacing $\lambda$ and propagation velocity $C_{w}$ can be approximately treated independently of each other. Based on physical analyses, both of them are dependent on the five physical qualities, that is, $\Gamma, \rho, v, \sigma, g \sin \beta$. Thus, there are six variables in three dimensions, that is, $\mathrm{m}, \mathrm{s}$, and $\mathrm{kg}$, for both primary pulse separation and propagation velocity in film flow. These variables can be combined into three dimensionless groups to correlate primary pulse spacing and propagation velocity. Four dimensionless groups are derived in the same way as Koizumi et al. [12]:

(a) Nondimensional propagation velocity

$$
N_{\text {uw }}=\frac{C_{w}}{(\nu g \sin \beta)^{1 / 3}} .
$$

(b) Nondimensional primary pulse spacing

$$
N_{\lambda}=\frac{\lambda}{h_{\mathrm{Nu}}} \text {. }
$$

(c) Film Reynolds number

$$
\operatorname{Re}=\frac{\Gamma}{\mu} .
$$

(d) Froude number

$$
\mathrm{Fr}=\frac{C_{w}}{\sqrt{g \sin \beta h_{\mathrm{Nu}}}} .
$$

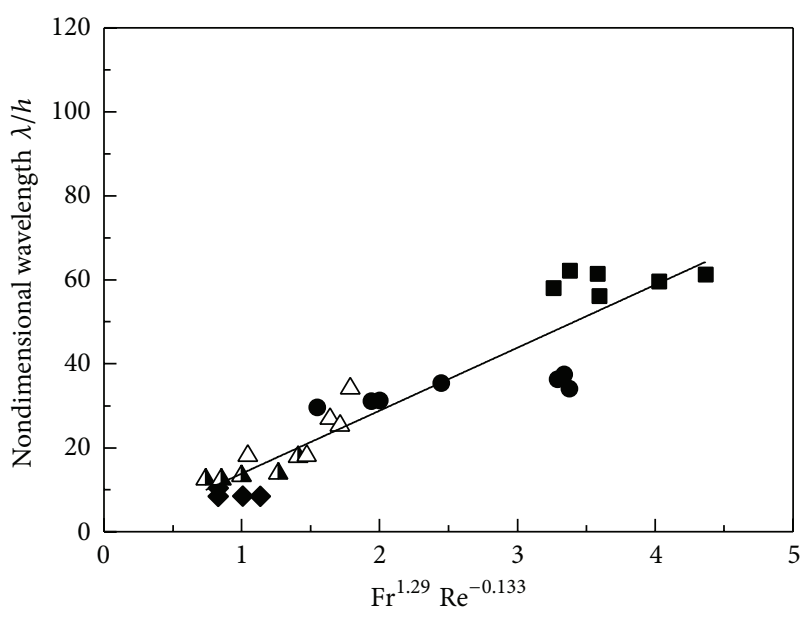

Present work

- Water at incli. $8^{\circ}$

- Water at incli. $13^{\circ}$

- Water at incli. $21^{\circ}$
Koizumi work

$\Delta$ Water on vertical wall 1999

$\triangle$ Water on vertical wall 2009

- Koizumi correlation
FIGURE 8: Comparison of nondimensional primary pulse spacing between experiments and Koizumi correlation.

The present data of primary pulse spacing and propagation velocity are reformulated with the film Reynolds number and the Froude number using the Koizumi nondimensional correlations, as shown in (6) and (7). Comparing with other relevant correlations, Koizumi correlation is a closure relation under the inlet boundary conditions, which requires only the film flow rate given at the inlet, the geometrical parameters of film plate, and the physical properties of working fluid.

$$
\begin{aligned}
N_{\lambda} & =12.39 \operatorname{Fr}^{1.29} \operatorname{Re}^{-0.133}, \\
N_{\text {uw }} & =1.13 \mathrm{Fr}^{0.582} \operatorname{Re}^{0.429} .
\end{aligned}
$$

Figure 8 shows the present data of nondimensional primary pulse spacing. The Koizumi data with water are also presented in Figure 8 for comparison. The continuous line is correlation (6). The deviation of all the present data from the correlation falls into the $\pm 20 \%$ offset strip.

The present data of nondimensional propagation velocity are shown in Figure 9. The data of Koizumi et al. [12] for water film on the outer wall of a vertical pipe and the data of Takahama and Kato [16] for the water film on the inner wall of a vertical pipe are also plotted in the figure. The continuous line is correlation (7). The deviation of all the present data from the correlation falls into the $\pm 15 \%$ offset strip.

3.2. Transition to Three-Dimensional Disordered Wave Pattern. The experimental observations show that two-dimensional regular solitary wave pattern is in anstable state and eventually destructed into three-dimensional disordered wave pattern. It is supposed that there are certainly some fundamental instabilities which trigger the process at some critical conditions. On the destruction mechanism, previous researchers had striven to elucidate it based on some hypotheses. Park and Nosoko [10], Demekhin et al. [17], and Kofman 


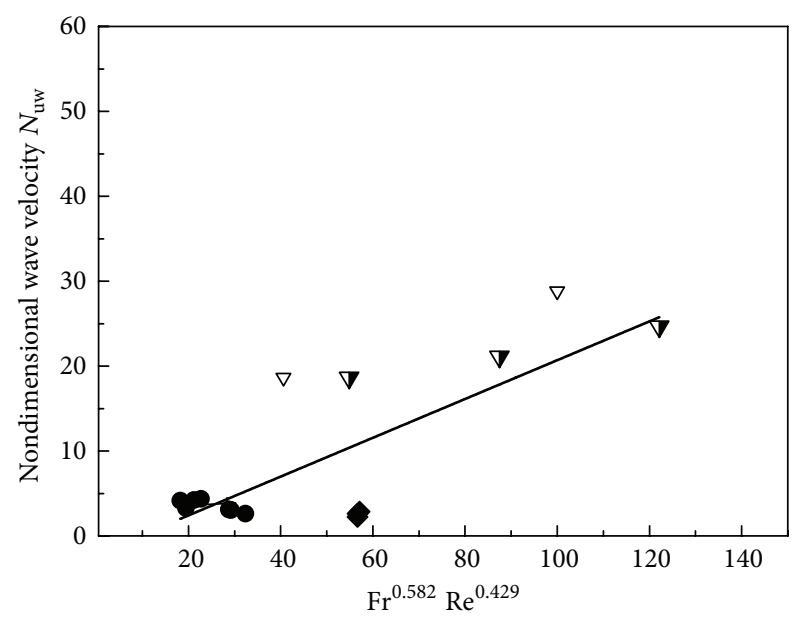

Present work

- Water at $8^{\circ}$
- Water at $13^{\circ}$
Water at $21^{\circ}$

$\nabla \quad$ Koizumi water 2009

$\nabla$ Takahama water 1980

— Koizumi correlation

FIgURE 9: Comparison of nondimensional propagation velocity between experiments and Koizumi correlation.

et al. [18] attributed it to the Plateau-Rayleigh instability, which is assumed to occur on the steep ridge between primary solitary pulse and its immediate preceding capillary ripple due to high concentration of surface tension force. And Kofman et al. [18] thought that the Plateau-Rayleigh instability only has effect on the transition in a capillary region with short-wave mode whereas for long-wave mode the transition is triggered by the Rayleigh-Taylor instability. Liu et al. [3] and Scheid et al. [19] ascribed it to 3D synchronous transverse modulations along the trough of primary solitary pulse, but the mechanism behind it was not yet illuminated.

Our experiments show that the transition to threedimensional disordered wave pattern from regular twodimensional solitary wave pattern is a fleeting process, which is initiated on the wave-front of primary solitary pulse, and then it precipitately breaks up into discontinuous irregular patterns with different size and shape; that is, some are like caterpillar and some are like crater, as shown in Figure 10. The most compelling feature of three-dimensional wave pattern is that the broken wave segments are with different size and shape and are being evolved out of phase for successive wave fronts, which are originally in phase and separated from one another regularly. Such the patterns and sizes formed are obviously different from that under the PlateauRayleigh instability, the typical pattern under which is equally distributed bead-like with similar size and shape.

Through the scrutiny of the transition process from the present experiments, it is found out that bubble-spike structure is firstly formed on the steep front of solitary pulse before the breakup of two-dimensional solitary structure. The bubble-spike structure on interface between fluids is typical of the Rayleigh-Taylor instability under gravity field. Triggering the Rayleigh-Taylor instability on the solitary pulse front, we think, is supposed to be due to steepness of

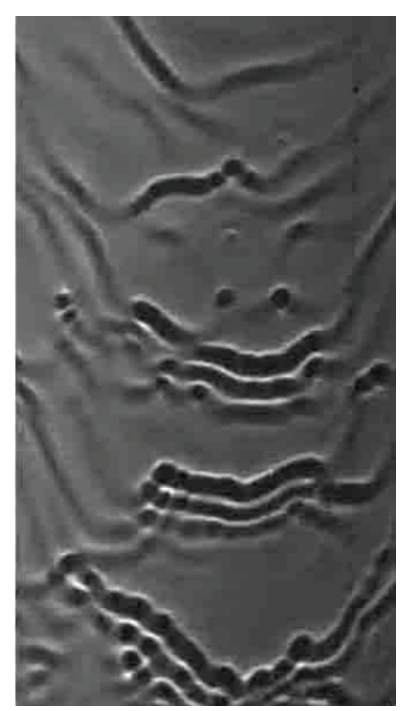

FIgURE 10: Three-dimensional irregular wave pattern.

solitary pulse front, which is almost vertical to the film plate. Under the stream-wise component of gravity, the RayleighTaylor instability is induced on the front surface of solitary pulse. In addition, the present experiments show that the time scale of the transition process is also consistent with characteristic time scale of the Rayleigh-Taylor instability.

\section{Conclusions}

Wave characteristics of falling film on inclination plate at the moderate Reynolds number are experimentally investigated using shadowgraphy, the spatial evolution scenario of which covers from initial disturbance up to transition to threedimensional irregular wave pattern. Working fluid is water at the room temperature and atmospheric pressure. The following conclusions are achieved:

(1) At the moderate Reynolds number, the evolution of surface disturbance in thin film flow under the natural excitation manifests itself clearly by three distinctive evolution regimes along stream-wise direction, namely, initial quiescent smooth film flow, twodimensional regular solitary wave pattern riding on film flow, and three-dimensional irregular wave pattern formed on the surface of film flow.

(2) The inception length of sensible two-dimensional solitary wave pattern is more dependent on the inclination angle than the Reynolds number at the moderate Reynolds number. It is inferred that wave evolution on film flow at the moderate Reynolds number is controlled by gravity and drag.

(3) On the development of two-dimensional regular solitary wave pattern, the two parameters, namely, primary pulse spacing and propagation velocity, which are significant to engineering applications for estimating heat and mass transfer on film flow, are investigated. The present experimental results of the 
two parameters are compared with the Koizumi correlations, which is the closed correlations under natural excitation. The deviation of the present experimental data from the correlations is limited to $20 \%$ and $15 \%$, respectively, for primary pulse spacing and propagation velocity.

(4) On the transition from two-dimensional regular solitary wave pattern to three-dimensional irregular wave pattern, it is revealed that the disintegration of continuous two-dimensional regular solitary wave pattern is triggered by the Rayleigh-Taylor instability that occurred on the steep front of primary pulse. This is in disagreement with other researchers, for example, the Plateau-Rayleigh instability and 3D synchronous moderation.

\section{Nomenclature}

$C_{w}:$ Propagation velocity

Fr: Froude number

$h_{\mathrm{Nu}}$ : Nusselt flat film thickness

g: Gravitational acceleration

$N_{\text {uw }}$ : Nondimensional propagation velocity

$N_{\lambda}$ : Nondimensional primary pulse spacing

Re: Film Reynolds number

$V_{\mathrm{Nu}}$ : Nusselt mean film velocity

$\beta$ : $\quad$ Angle between film plate and horizontal direction

$\lambda$ : $\quad$ Primary pulse spacing

$\mu$ : Dynamic viscosity

$\rho:$ Density.

\section{Competing Interests}

The authors declared no potential conflict of interests with respect to the research, authorship, and/or publication of this article.

\section{Acknowledgments}

The authors disclosed receipt of the following financial support for the research, authorship, and/or publication of this article: This paper is based on some of the results of projects under Grant nos. 51321002 and 11472155, which were supported by the Science Fund for Creative Research Groups of the National Natural Science Foundation of China.

\section{References}

[1] Y. Q. Yu and X. Cheng, "Experimental study of water film flow on large vertical and inclined flat plate," Progress in Nuclear Energy, vol. 77, pp. 176-186, 2014.

[2] S. Portalski, "Studies of falling liquid film flow film thickness on a smooth vertical plate," Chemical Engineering Science, vol. 18, no. 12 , pp. 787-804, 1963.
[3] J. Liu, J. B. Schneider, and J. P. Gollub, "Three-dimensional instabilities of film flows," Physics of Fluids, vol. 7, no. 1, pp. 5567, 1995.

[4] T. Nosoko, P. N. Yoshimura, T. Nagata, and K. Oyakawa, "Characteristics of two-dimensional waves on a falling liquid film," Chemical Engineering Science, vol. 51, no. 5, pp. 725-732, 1996.

[5] P. L. Kapitza and S. P. Kapitza, "Wave flow of thin fluid layers of liquid," in Collected Papers of P. L. Kapitza, D. T. Haar, Ed., pp. 662-709, Pergamon Press, Oxford, UK, 1964.

[6] J. Liu and J. P. Gollub, "Solitary wave dynamics of film flows," Physics of Fluids, vol. 6, no. 5, pp. 1702-1712, 1994.

[7] J. Liu, J. D. Paul, and J. P. Gollub, "Measurements of the primary instabilities of film flows," Journal of Fluid Mechanics, vol. 250, pp. 69-101, 1993.

[8] K. J. Chu and A. E. Dukler, "Statistical characteristics of thin, wavy films: part II. Studies of the substrate and its wave structure," AIChE Journal, vol. 20, no. 4, pp. 695-706, 1974.

[9] R. P. Salazar and E. Marschall, “Time-average local thickness measurement in falling liquid film flow," International Journal of Multiphase Flow, vol. 4, no. 4, pp. 405-412, 1978.

[10] C. D. Park and T. Nosoko, "Three-dimensional wave dynamics on a falling film and associated mass transfer," AIChE Journal, vol. 49, no. 11, pp. 2715-2727, 2003.

[11] L. O. Jones and S. Whitaker, "An experimental study of falling liquid films," AIChE Journal, vol. 12, no. 3, pp. 525-529, 1966.

[12] Y. Koizumi, R. Enari, and H. Ohtake, "Correlations of wave characteristics for a liquid film falling down along a vertical wall," Journal of Heat Transfer, vol. 131, no. 8, pp. 1-9, 2009.

[13] M. J. Lighthill, Waves in Fluids, Cambridge University Press, Cambridge, UK, 2nd edition, 2001.

[14] V. P. Carey, Liquid-Vapor Phase-Change Phenomena, Hemisphere Publishing Corporation, Bristol, UK, 1992.

[15] J. H. Lienhard IV and J. H. Lienhard V, A Heat Transfer Textbook, Phlogiston Press, Cambridge, UK, 3rd edition, 2008.

[16] H. Takahama and S. Kato, "Longitudinal flow characteristics of vertically falling liquid films without concurrent gas flow," International Journal of Multiphase Flow, vol. 6, no. 3, pp. 203215, 1980.

[17] E. A. Demekhin, E. N. Kalaidin, S. Kalliadasis, and S. Y. Vlaskin, "Three-dimensional localized coherent structures of surface turbulence. I. Scenarios of two-dimensional-three-dimensional transition," Physics of Fluids, vol. 19, no. 11, Article ID 114103, 2007.

[18] N. Kofman, S. Mergui, and C. Ruyer-Quil, "Three-dimensional instabilities of quasi-solitary waves in a falling liquid film," Journal of Fluid Mechanics, vol. 757, pp. 854-887, 2014.

[19] B. Scheid, C. Ruyer-Quil, and P. Manneville, "Wave patterns in film flows: modelling and three-dimensional waves," Journal of Fluid Mechanics, vol. 562, pp. 183-222, 2006. 

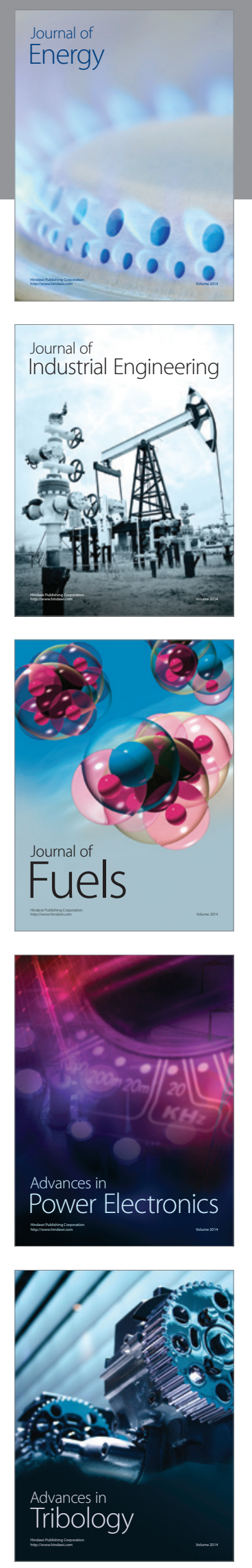
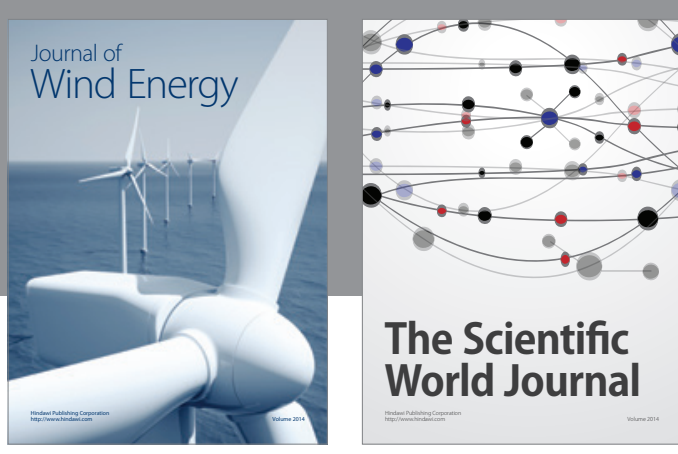

The Scientific World Journal
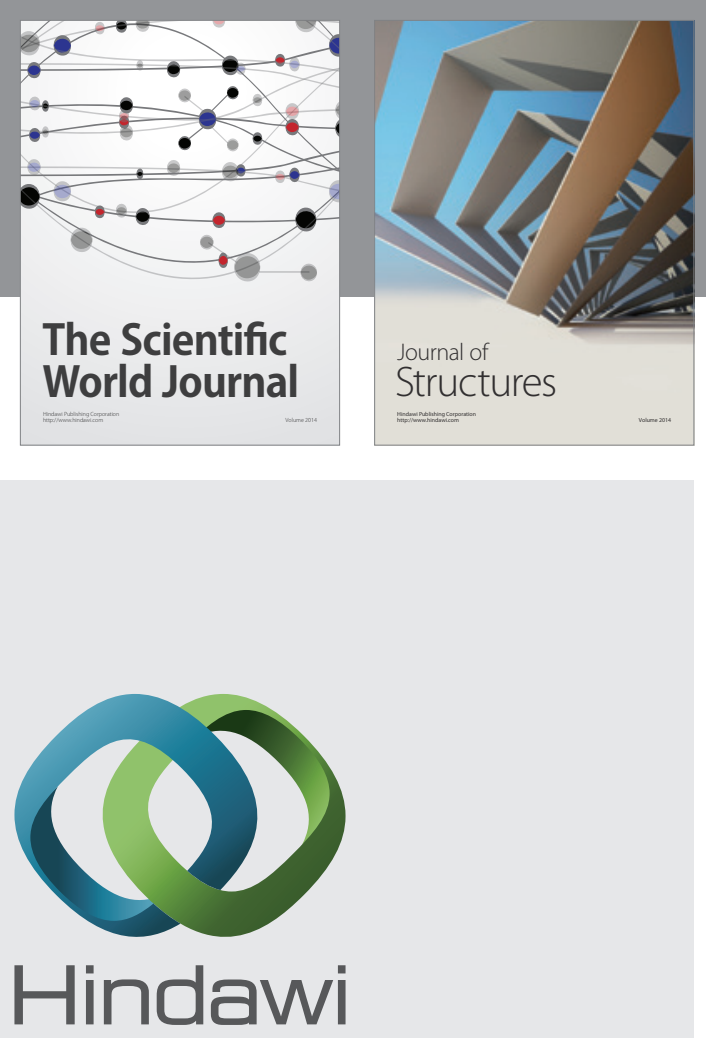

Submit your manuscripts at

http://www.hindawi.com
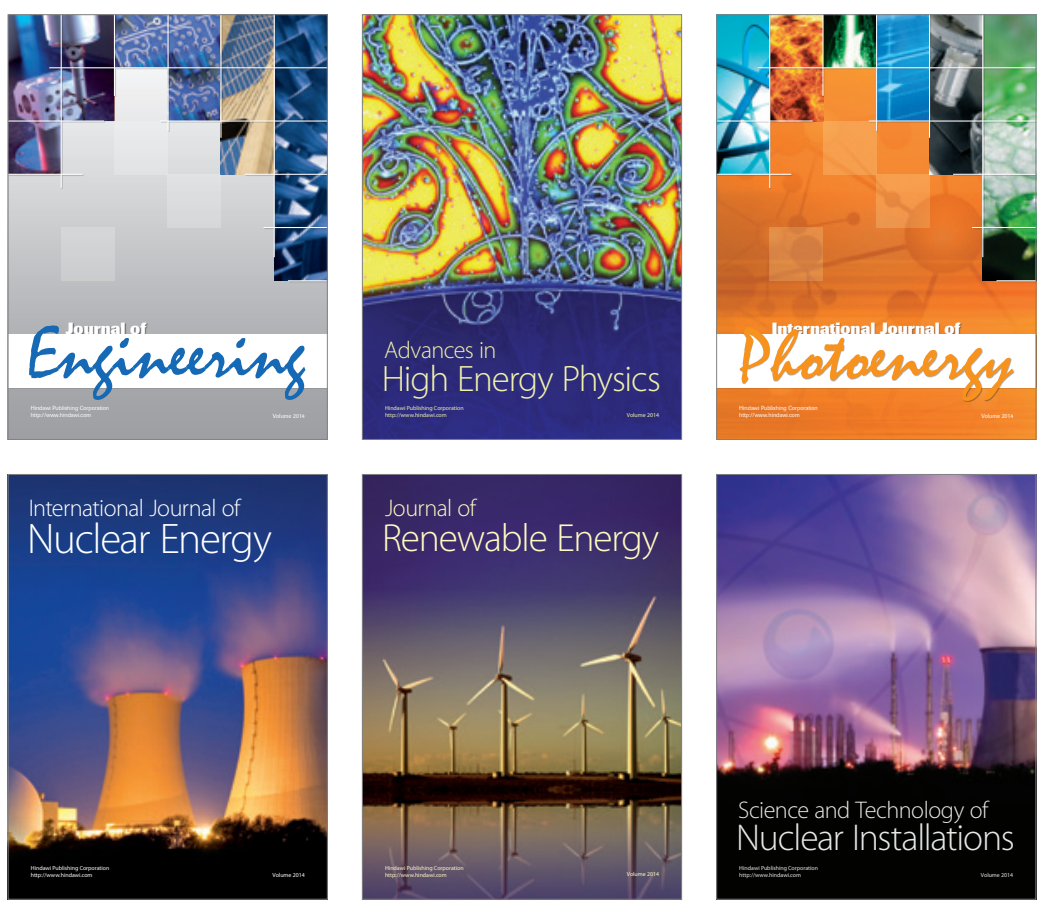
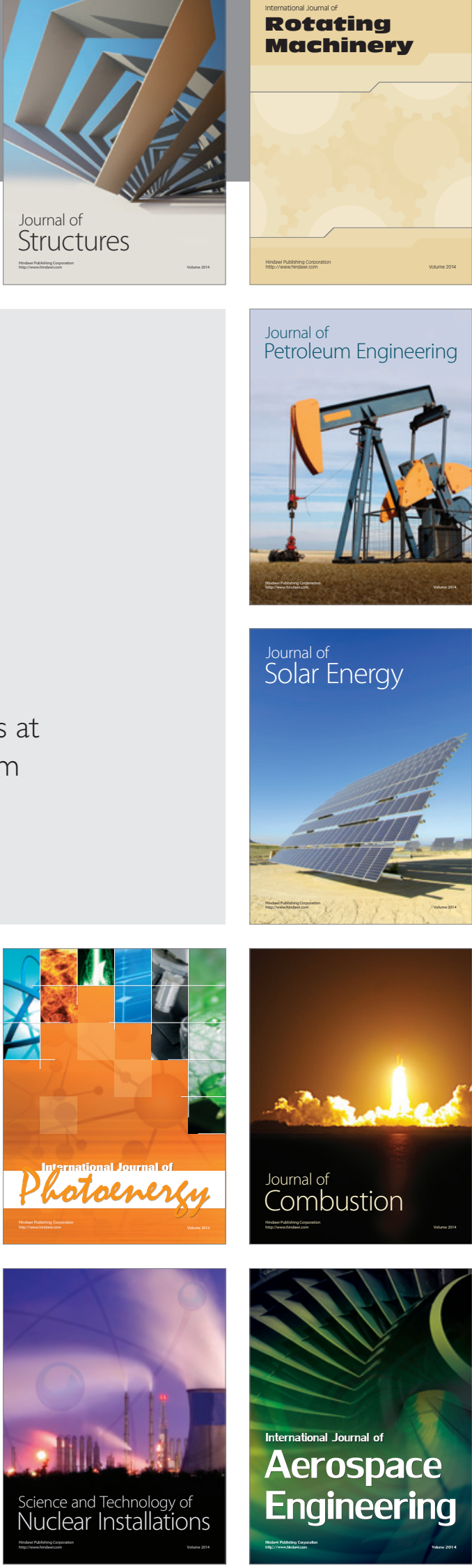